\title{
Comparison of FitBit ${ }^{\circledR}$ Ultra to ActiGraphTM GT1M for Assessment of Physical Activity in Young Adults During Treadmill Walking
}

\author{
R.J. Gusmer, T.A. Bosch ${ }^{*}$, A.N. Watkins, J.D. Ostrem and D.R. Dengel
}

School of Kinesiology, University of Minnesota, 1900 University Avenue SE, Minneapolis, MN 55455, USA

\begin{abstract}
Introduction: Accelerometers are used to quantify energy expenditure in field research. The ActiGraph ${ }^{\mathrm{TM}}$

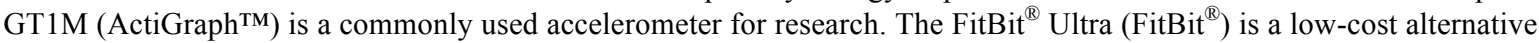
to the ActiGraph ${ }^{\mathrm{TM}}$; however, there is limited research on the validity of this device.

Purpose: The pilot study compares the FitBit ${ }^{\circledR}$ against the ActiGraph ${ }^{\mathrm{TM}}$ and metabolic cart for measurement of energy expenditure and step counts during treadmill walking.

Methods: Thirty-two ( 25 female) adults, mean age $22 \pm 2$ years, performed two thirty-minute phases of walking (slow and brisk) on a treadmill while concurrently wearing the FitBit ${ }^{\mathbb{B}}$ and the ActiGraph ${ }^{\mathrm{TM}}$. Energy expenditure estimates were compared against energy expenditure measured by a metabolic cart. The Pearson's correlation and t-tests determine the linear association and similarity between the accelerometers.

Results: Energy expenditure estimate is moderately correlated between the two accelerometers during slow walking $(\mathrm{r}=0.584, p=0.011)$ and strongly correlated during brisk walking $(\mathrm{r}=0.910, p<0.001)$. Step count is strongly correlated between the accelerometers during slow $(\mathrm{r}=0.974, p<0.001)$ and brisk $(\mathrm{r}=0.996, p<0.001)$ walking. The FitBit ${ }^{\circledR}$ significantly underestimated energy expenditure during brisk walking compared to metabolic cart data. There is no difference between the slow and brisk phases' step counts using either accelerometer.

Conclusion: The results of this pilot study suggest that the FitBit ${ }^{\circledR}$ and the ActiGraph ${ }^{\mathrm{TM}}$ can be used interchangeably to measure steps, but not to measure kilocalories. Furthermore, the FitBit ${ }^{\circledR}$ underestimates energy expenditure, compared to a metabolic cart, as exercise intensity increases. This limits its ability to accurately measure energy expenditure in active populations.
\end{abstract}

Keywords: Accelerometer, Bland-Altman, exercise, hysical activity, obesity, validation, walking.

\section{INTRODUCTION}

Currently, the prevalence of obesity in the United States is approximately $35 \%$ among adults and $17 \%$ among adolescents $[1,2]$. Obesity is associated with serious health complications such as type 2 diabetes, cardiovascular disease, and the metabolic syndrome [3]. Physical activity is as an intrinsic part of combating obesity and achieving a healthy lifestyle [4]. Monitoring physical activity is important for assessing activity patterns in populations being studied; however, even more important is the validity of the data being collected $[5,6]$. Without a valid measurement, it is difficult to assess treatment outcomes.

Accelerometers are devices that detect movement and can estimate the user's step count and energy expenditure using standardized equations [5]. The ActiGraph ${ }^{\mathrm{TM}}$ (ActiGraph $^{\mathrm{TM}}$, Pensacola, Florida, USA) is one of the most commonly used accelerometers in research [7]. The ActiGraph $^{\mathrm{TM}}$ provides information regarding step counts, intensity levels, energy expenditure, and sleep measurements. The ActiGraph ${ }^{\mathrm{TM}}$ is a valid and reliable

*Address correspondence to this author at the 1900 University Ave SE, Minneapolis, MN 55455, USA; Tel: 612-236-6457; Fax: 1-612-625-5300; E-mail: bosch041@umn.edu device in several populations [7-11]; however, there is a need in research for a more feasible option for monitoring physical activity in the general population. Recently, the FitBit $^{\circledR}$ Ultra, (FitBit ${ }^{\circledR}$ Inc., San Francisco, California, USA) was developed as a commercially available accelerometer. This new device is lower in cost and smaller in size than the ActiGraph ${ }^{\mathrm{TM}}$. Additionally, the $\mathrm{FitBit}^{\left({ }^{\circledR}\right.}$ uploads data wirelessly to a web-based database and provides direct feedback from the device to the user. If valid, this greatly increases the feasibility of data acquisition.

There is limited research on the how the FitBit ${ }^{\circledR}$ performs compared to other accelerometers or metabolic cart measurements. Comparison to other accelerometers is essential before the FitBit ${ }^{\circledR}$ is used for research. This pilot study compares the FitBit ${ }^{\mathbb{R}}$ against a widely used instrument, the ActiGraph ${ }^{\mathrm{TM}}$, during treadmill walking in young adults. Both accelerometers are compared against energy expenditure measured by metabolic cart.

\section{METHODS}

\section{Participants}

The University of Minnesota Institutional Review Board (IRB) approved all procedures. Participants were recruited from students at the University of Minnesota-Twin Cities 
campus. Thirty-two participants between 18-29 years old (mean 22 \pm 2 years) performed two 30-minute phases of walking at slow and brisk speeds on a treadmill while concurrently wearing the FitBit ${ }^{\circledR}$ and ActiGraph ${ }^{\mathrm{TM}}$ GT1M. Additionally, participants wore a neoprene facemask (New Leaf, St. Paul, MN) connected to a CPX Ultima ${ }^{\circledR}$ metabolic cart (Medgraphics ${ }^{\circledR}$, Saint Paul, MN, USA). Five participants were excluded because the ActiGraph ${ }^{\mathrm{TM}}$ failed to correctly measure step counts (counts were half the value measured by FitBit $\left.^{\circledR}\right)$. Six participants were excluded for missing metabolic cart data. Data from twenty-one participants is used for comparison (Table 1).

Table 1. Descriptive Statistics of Participants (Mean \pm SD)

\begin{tabular}{|l|c|c|c|}
\hline \multicolumn{1}{|c|}{ Variable } & $\begin{array}{c}\text { Female } \\
(\boldsymbol{n}=\mathbf{2 5})\end{array}$ & $\begin{array}{c}\text { Male } \\
(\boldsymbol{n}=\mathbf{7})\end{array}$ & $\begin{array}{c}\text { Total } \\
(\boldsymbol{n}=\mathbf{3 2})\end{array}$ \\
\hline \hline Age (yrs) & $21.2 \pm 1.3$ & $21.3 \pm 3.5$ & $21.2 \pm 1.7$ \\
\hline Height (cm) & $165.9 \pm 5.5$ & $174.4 \pm 5.1$ & $167.1 \pm 6.1$ \\
\hline Weight (kg) & $57.9 \pm 6.8$ & $83.3 \pm 19.5$ & $61.5 \pm 12.7$ \\
\hline Race (\% white) & $94 \%$ & $33 \%$ & $86 \%$ \\
\hline Step Length (m) $(n=6)$ & $0.65 \pm 0.04$ & $0.77 \pm 0.02$ & $0.68 \pm 0.07$ \\
\hline Slow Walking Speed (m/s) & $1.09 \pm 0.12$ & $1.21 \pm 0.13$ & $1.11 \pm 0.12$ \\
\hline Brisk Walking Speed $(\mathrm{m} / \mathrm{s})$ & $1.34 \pm 0.15$ & $1.46 \pm 0.13$ & $1.36 \pm 0.16$ \\
\hline
\end{tabular}

\section{Data Collection and Procedures}

Following informed consent, demographic information was recorded. Height was measured (to the nearest $\mathrm{cm}$ ) using a stadiometer. Body mass was determined (to the nearest 0.1 $\mathrm{kg}$ ) using an electronic scale. Step length was established by having each participant take twenty strides. The distance was measured to the nearest half inch and divided by the number of strides to get the average stride length [12]. The FitBit ${ }^{\circledR}$ was updated with the subject's height, weight, and step length. Prior to testing, the ActiGraph ${ }^{\mathrm{TM}}$ G1TM was initialized and set to collect data on two axes at 60-second epoch rates to match the minute-by-minute data collection the FitBit ${ }^{\circledR}$ Ultra.

The FitBit ${ }^{\circledR}$ was placed on the midaxillary line of the subject's right side using the FitBit $^{\left({ }^{B}\right.}$ holster. The ActiGraph $^{\mathrm{TM}}$ was secured on the midaxillary line of the participant's left side using an elastic belt. Participants were fitted with a Polar heart rate monitor (Polar Electro Inc., Lake Success, NY, USA) and a neoprene facemask connected to a CPX Ultima $^{\circledR}$ metabolic cart for gas collection and analysis (Breezesuite, Medgraphics ${ }^{\circledR}$, Saint Paul, MN, USA).

\section{Calculation of Walking Speed}

Each participant was instructed how to safely get on and off the moving treadmill. Participants self-selected a comfortable walking speed by adjusting the treadmill speed in $0.089 \mathrm{~m} / \mathrm{s}$ increments. Slow and brisk pace was calculated as $\pm 10 \%$ of their self-selected comfortable walking speed (i.e. if pace was selected at $1.34 \mathrm{~m} / \mathrm{s}$, then slow $=1.21 \mathrm{~m} / \mathrm{s}$ and brisk $=1.46 \mathrm{~m} / \mathrm{s}$ ). A $10 \%$ change was used to measure each device's sensitivity to change, and was small enough to ensure that participants refrained from jogging.
Each walking phase lasted 30 minutes. Heart rate, oxygen uptake $\left(\mathrm{VO}_{2}\right)$, carbon dioxide production $\left(\mathrm{VCO}_{2}\right)$, ventilation $\left(\mathrm{V}_{\mathrm{E}}\right)$, and RER were collected at baseline and at two-minute intervals with data recorded from the last 10 seconds of every two minutes. The first phase consisted of slow walking. Following the first phase, participants rested until heart rate recovered within 10 beats of resting heart rate. Participants completed the brisk walking phase with the same measurements obtained during the last ten seconds of two-minute intervals.

\section{FitBit $^{\circledR}$ Data Collection for Analysis}

Following testing, the FitBit ${ }^{\circledR}$ data were transferred into minute-by-minute sample rate estimations for kilocalories and steps in order to compare with the ActiGraph ${ }^{\mathrm{TM}}$. The minute-by-minute code was acquired from the developers of FitBit $^{\circledR}$ to extract information from the FitBit ${ }^{\circledR}$ database, and an application programming interface (API) was used to convert the information. Data was extracted from the FitBit ${ }^{\circledR}$ using the API, providing minute-by-minute estimations of kilocalories and steps. The data were summed to produce a total estimate for each walking phase. To control for timing differences between the accelerometers, the first and last two minutes of each walking phase were excluded.

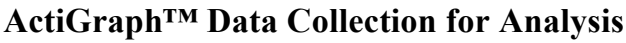

The ActiGraph ${ }^{\text {TM }}$ data were downloaded using ActiLife 5 software (ActiGraph ${ }^{\mathrm{TM}}$, LLC, Pensacola, FL, USA). Biometric information was inputted. The Freedson equation was used to estimate energy expenditure and steps counts [13]. Data was calculated in 60-second epochs to measure activity counts per minute. Standard axis counts per minute cut points from ActiLife 5 software were used and set as sedentary (0-99), light (100-759), lifestyle (760-1951), moderate (1952-5724), vigorous (5725-9498), and very vigorous [9499-infinity]. Activities within this study were categorized as sedentary, light, and lifestyle. Steps and kilocalories were calculated in one-minute estimates and summed to obtain a total estimate for each walking phase. To control time differences between accelerometers, the first and last two minutes of each phase were excluded. The ActiGraph $^{\mathrm{TM}}$ measures energy expenditure independent of resting energy expenditure. The ActiGraph ${ }^{\mathrm{TM}}$ energy expenditure estimate was adjusted to include the predicted resting energy expenditure in order to be comparable to the FitBit $^{B}$ and the metabolic cart calculations. These measurements include resting energy expenditure in their activity estimates.

\section{Calculation of Energy Expenditure from Metabolic Cart Data}

Data from the metabolic cart was used to estimate energy expenditure from two-minute averages of $\mathrm{VO}_{2}, \mathrm{RER}$, and energy expenditure $/ \mathrm{LO}_{2}$. These averages were totaled to obtain the average energy expenditure during each thirtyminute phase. The first and last two minutes were excluded to coincide with the FitBit ${ }^{\circledR}$ and ActiGraph ${ }^{\mathrm{TM}}$ data.

\section{Statistical Analysis}

Pearson's correlations were completed to examine the linear association between estimates of kilocalories and step counts given by the FitBit ${ }^{(B)}$ Ultra, the ActiGraph ${ }^{\mathrm{TM}}$ GT1M, 
and the metabolic cart measurements. Paired t-tests identify statistical differences between methods. The Bland-Altman [14] concordance technique was used to determine agreement between step counts and energy expenditure measurements by the FitBit ${ }^{\circledR}$ Ultra, ActiGraph ${ }^{\mathrm{TM}}$ and the metabolic cart. Agreement was determined by plotting the differences between the two accelerometers against their means and observing the spread and deviation of the data. Data are analyzed using R statistical analysis software (R Foundation for Statistical Computing, Vienna, Austria).

\section{RESULTS}

Table 1 presents participant characteristics, including mean step length and walking speeds. The values are separated by gender, but no tests for significance were conducted to compare these values.

\section{Energy Expenditure via Kilocalories}

During the brisk walking phase, all three measurements of energy expenditure are highly correlated. However, during slow walking the strongest correlation is between the ActiGraph ${ }^{\mathrm{TM}}$ and the metabolic cart measurements. The $\mathrm{FitBit}^{\circledR}$ is moderately correlated with the other measurements of energy expenditure during slow walking. Correlations for energy expenditure during slow and brisk walking are $\mathrm{r}=0.584 \quad(p=0.011)$ and $\mathrm{r}=0.910 \quad(p<0.001)$, respectively, between the ActiGraph ${ }^{\mathrm{TM}}$ and the $\mathrm{FitBit}^{\mathrm{B}}{ }^{\text {Ultra. The FitBit }}{ }^{\mathrm{B}}$ and the metabolic cart are moderately correlated $(\mathrm{r}=0.689$, $\mathrm{p}<0.001)$ during slow walking, and strongly correlated $(\mathrm{r}=0.942, \mathrm{p}<0.001)$ during brisk walking. The ActiGraph ${ }^{\mathrm{TM}}$ and the metabolic cart are strongly correlated at both slow $(\mathrm{r}=0.812, p<0.001)$ and brisk $(\mathrm{r}=0.918, p<0.001)$ walking speeds.

Table 2 presents the estimates of mean energy expenditure and step counts for each method. No differences exist between the accelerometers for energy expenditure estimates during the slow walking phase; however, compared to the metabolic cart measurements, the FitBit ${ }^{\mathbb{B}}$ significantly underestimates energy expenditure during brisk walking. No difference between the ActiGraph ${ }^{\mathrm{TM}}$ and the metabolic cart is observed during brisk walking. This suggests that as exercise intensity increases, the $\mathrm{FitBit}^{\mathbb{B}}$ underestimates kilocalories expended. Energy expenditures measured during slow walking speeds are significantly lower than during brisk walking for each method $(p<0.001)$. This suggests that each method detected a change in intensity between walking phases. The median differences between

Table 2. Means Table for Energy Expenditure and Steps by Device (Mean \pm SD)

\begin{tabular}{|c|c|c|c|}
\hline & $\begin{array}{l}\text { ActiGraph }^{\mathrm{TM}} \\
\text { GT1M }\end{array}$ & $\begin{array}{c}\text { FitBit }^{\circledR} \\
\text { Ultra }\end{array}$ & $\begin{array}{c}\text { Metabolic } \\
\text { Cart }\end{array}$ \\
\hline Slow Walking (kcals) & $89.2^{\mathrm{a} *} \pm 8.7$ & $88.0^{\mathrm{a} *} \pm 3.9$ & $100.9^{\mathrm{a}} \pm 5.8$ \\
\hline Brisk Walking (kcals) & $122.6^{\mathrm{ab} *} * 11.6$ & $100.9^{b * \pm 6.3}$ & $121.9^{\mathrm{a}} \pm 7.7$ \\
\hline Slow Walking (Steps/min) & $105.3^{\mathrm{a} *} \pm 6.5$ & $105.9^{\mathrm{a} *} \pm 5.8$ & - \\
\hline Brisk Walking (Steps/min) & $114.2^{\mathrm{a} *} \pm 5.6$ & $113.9^{a *} \pm 5.2$ & - \\
\hline
\end{tabular}

Differences between means for each row are denoted by a difference in letter next to the mean at $(p<0.05)$.

*Signifies a difference $(p<0.05)$ between slow and brisk walking measurements. accelerometers at slow and brisk speeds are $23 \mathrm{kcals} / \mathrm{min}$ and $10 \mathrm{kcals} / \mathrm{min}$, respectively. Bland-Altman plots for the accelerometers show average agreement but high variability (Figs, 1A, B). Points above zero on the Bland-Altman plot indicate higher measured kilocalories by the ActiGraph ${ }^{\mathrm{TM}}$ GT1M, while points below zero indicate higher measured values by the FitBit ${ }^{\circledR}$ Ultra. The Bland-Altman plots for the $\mathrm{FitBit}^{\mathbb{B}}$ and the metabolic cart demonstrate average agreement but a large deviation in the difference between measurements (Fig. 1C, D).

\section{Step Counts}

Average step counts are presented in Table 2. Step counts are not significantly different between the accelerometers $(p=0.892)$. Step counts during slow walking are significantly lower $(p<0.001)$ than during brisk walking for both accelerometers. This indicates a significant difference in walking speed. Step counts between each accelerometer are strongly correlated during slow $(\mathrm{r}=0.974, p<0.001)$ and brisk $(\mathrm{r}=0.996, p<0.001)$ walking. The $95 \%$ confidence intervals at slow and brisk speeds are [0.935-0.990] and [0.988-0.998], respectively. Median differences between accelerometers at slow and brisk speeds are $3.0 \mathrm{steps} / \mathrm{min}$ and $1.0 \mathrm{steps} / \mathrm{min}$, respectively. Bland-Altman plots show strong agreement for both speeds. Six participants were excluded as their ActiGraph $^{\mathrm{TM}}$ step counts were half of the FitBit ${ }^{\circledR}$ step counts. This was likely an ActiGraph ${ }^{\mathrm{TM}}$ malfunction caused by a slow walking pace.

\section{DISCUSSION}

The present study observes that the $\mathrm{FitBit}^{\circledR}$ can be used interchangeably with the ActiGraph ${ }^{\mathrm{TM}}$ to measure step counts, but not for the estimation of energy expenditure. Furthermore, the FitBit ${ }^{\circledR}$ is capable of measuring exercise at two different walking speeds; however, it does appear to underestimate energy expenditure as compared to metabolic cart data. This could be a result of the method used by $\mathrm{FitBit}^{\mathbb{B}}$ to estimate energy expenditure. $\mathrm{FitBit}^{\mathrm{B}}$ provides the option of manually logging activity; it may be beneficial to compare estimates of energy expenditure between manually logged activities and automatic estimates of energy expenditure using $\mathrm{FitBit}^{\circledR}$ in future studies.

The linear relationship of energy expenditure is stronger during brisk walking. This is not surprising because the ActiGraph $^{\mathrm{TM}}$ has been identified as being less accurate during low intensities [7]. It is worth noting that despite device calculation differences within each person, the average energy expenditure for the entire sample was similar between accelerometers. Linearity and agreement improve during brisk walking, yet there was still a wide variation between the accelerometers. The variation is considerable for only 30 minutes of activity. During brisk walking, the ActiGraph $^{\text {TM }}$ provides a similar estimate of energy expenditure compared to the metabolic cart, whereas the $\mathrm{FitBit}^{\circledR}$ underestimated energy expenditure during brisk walking. These results raise questions about the ability of the $\mathrm{FitBit}^{\mathbb{B}}$ to accurately measure energy expenditure above slow walking. Without validation at increased exercise intensities, the FitBit ${ }^{\circledR}$ should not be used for research.

These accelerometers are much more comparable when measuring step counts. At both walking speeds, average step counts are very close. Additionally, the difference in step 
(A)

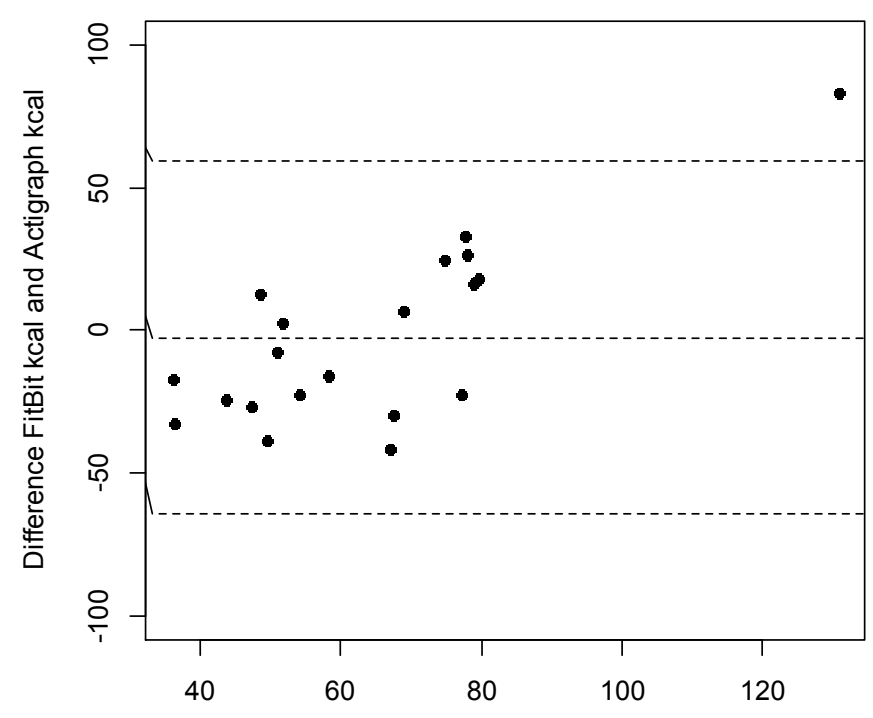

Average FitBit kcal and Actigraph kcal

(C)

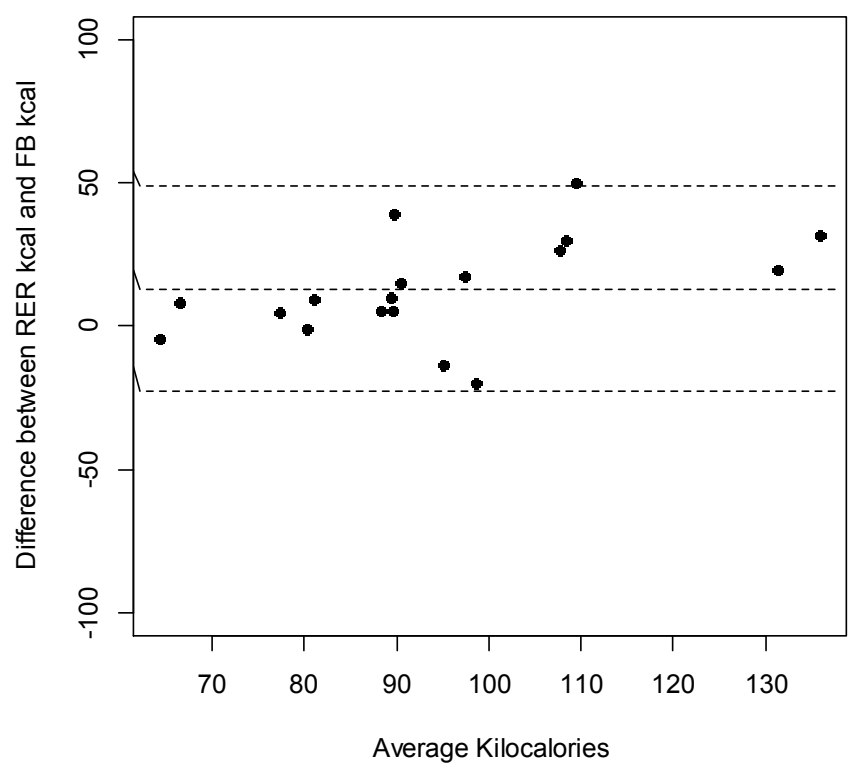

(B)

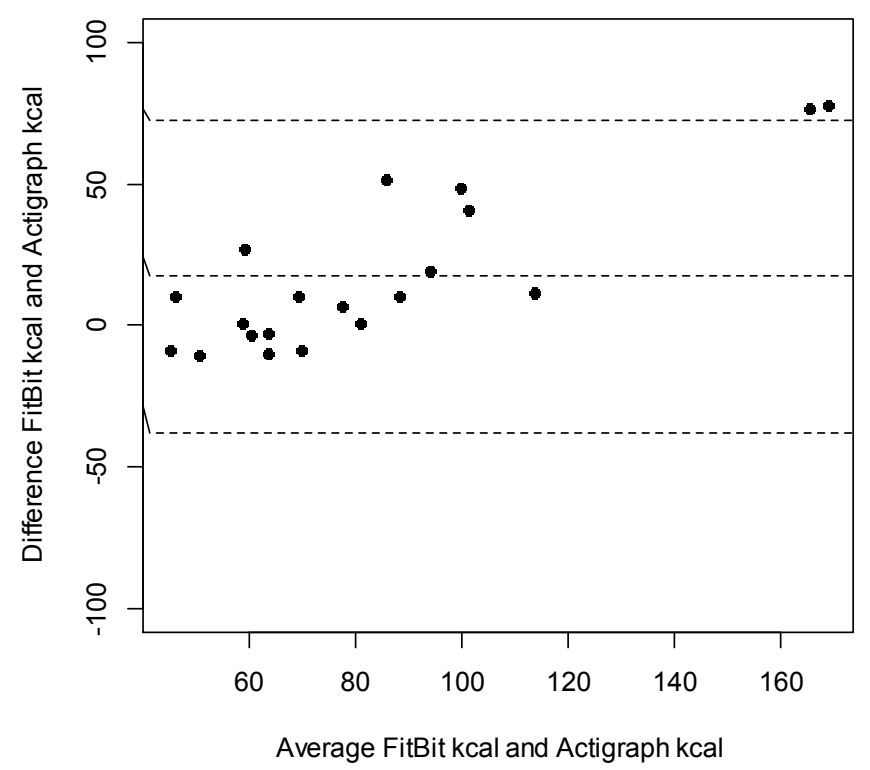

(D)

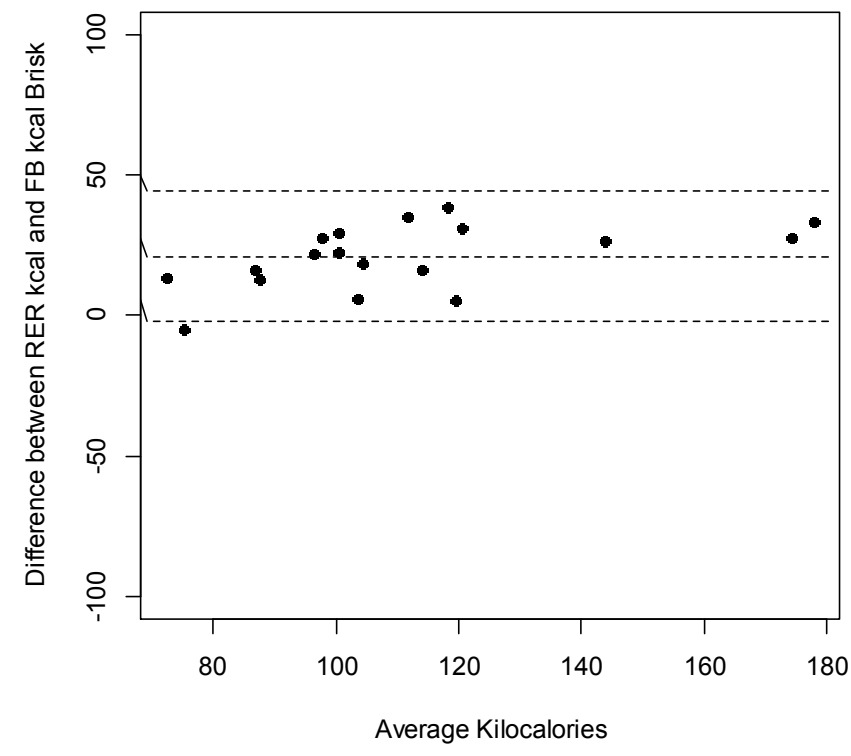

Fig. (1). Bland-Altman plot of kilocalories measured by each accelerometer during slow walking phase (A); kilocalories measured by each accelerometer during brisk walking phase $(\mathbf{B})$; kilocalories measured by FitBit ${ }^{\mathbb{B}}$ and metabolic cart for slow walking $(\mathbf{C})$; kilocalories measured by FitBit ${ }^{\circledR}$ and metabolic cart for Brisk Walking (D).

counts between each speed suggests that both accelerometers can detect small changes in gait speed during walking. This demonstrates these accelerometers' abilities to measure step counts. However, no comparison was made with actual steps taken, and thus we must rely on the similarity of each device to determine the accuracy of the measurement. It is important to note that six participants were excluded because their step counts as measured by the ActiGraph ${ }^{\mathrm{TM}}$ were half the values that were measured by the FitBit ${ }^{\circledR}$ Ultra. This may have been due to incorrect device placement or a lack of ActiGraph $^{\mathrm{TM}}$ sensitivity during slow walking. Therefore, the FitBit $^{\circledR}$ may be a more sensitive to motion at slow walking speeds, which may be conducive for public health research in elderly populations where movement is slower.
The results of this study indicate the FitBit $^{\circledR}$ and ActiGraph $^{\mathrm{TM}}$ have poor to average agreement when measuring energy expenditure, and strong agreement when measuring steps during slow and brisk walking on a treadmill. At slow speeds, they provide a similar population estimate of energy expenditure, but not individual estimates. As exercise intensity increases, the $\mathrm{FitBit}^{\circledR}$ underestimates energy expenditure compared to both the ActiGraph ${ }^{\mathrm{TM}}$ and the metabolic cart data. These finding suggests that these accelerometers should not be used interchangeably as they do not give similar estimates of kilocalories expended, and the FitBit $^{\circledR}$ may underestimate energy expenditure as exercise intensity increases. 


\section{CONCLUSION}

The ability of accelerometers to objectively quantify physical activity levels is important in promoting health. The FitBit $^{\circledR}$ and the ActiGraph ${ }^{\text {TM }}$ assist in objectively quantifying physical activity by providing quantitative measures of energy expenditure and steps. The results of this pilot study provide evidence that the $\mathrm{FitBit}^{\mathbb{B}}$ and the ActiGraph ${ }^{\mathrm{TM}}$ may be used interchangeably to measure steps, but not kilocalories. However, it should be noted that the ActiGraph ${ }^{\mathrm{TM}}$ did fail to accurately measure step counts in some participants during slow walking. The ActiGraph ${ }^{\mathrm{TM}}$ provides greater accuracy in assessment of varying intensities. This study illustrates the FitBit ${ }^{\circledR}$ underestimates energy expenditure during both slow and brisk walking compared to metabolic cart data. As such, the validity of the FitBit $^{\circledR}$ to accurately measure energy expenditure needs to be examined further before it is used in research.

\section{LIMITATIONS}

Limitations include the small sample and lack of diversity in the sample size. The small sample size likely led to a decrease in power. The walking trials took place in controlled laboratory conditions, which may differ from normal activities of living. Future studies are warranted to counter these limitations. Despite these limitations, this pilot study provided evidence of the usefulness of the $\mathrm{FitBit}^{\mathbb{B}}$ to measure step counts in future research. The validity and reliability of the FitBit ${ }^{\circledR}$ during running and other activities remains unknown.

\section{CONFLICT OF INTEREST}

The authors confirm that this article content has no conflict of interest.

\section{ACKNOWLEDGEMENTS}

The authors acknowledge and thank the participants for their time and effort. Funding and support for this research was provided by the University of Minnesota Undergraduate
Research Opportunities Program. The authors do not have any conflict of interests with this study.

\section{REFERENCES}

[1] Flegal KM, Carroll MD, Kit BK, Ogden CL. Prevalence of obesity and trends in the distribution of body mass index among US adults, 1999-2010. JAMA 2012: 307(5): 491-7.

[2] Ogden CL, Carroll MD, Kit BK, Flegal KM. Prevalence of obesity and trends in body mass index among US children and adolescents, 1999-2010. JAMA 2012: 307(5): 483-90.

[3] Grundy SM. Obesity, metabolic syndrome, and cardiovascular disease. J Clin Endocrinol Metabol 2004; 89(6): 2595-600.

[4] Ogden CL, Carroll MD, Curtin LR, McDowell MA, Tabak CJ, Flegal KM. Prevalence of overweight and obesity in the United States 1999-2004. JAMA 2006: 295:1549-55.

[5] Rothney MP, Schaefer EV, Neumann MN, Choi L, Chen KY. Validity of physical activity intensity predictions by ActiGraph, Actical, and RT2 accelerometers. Obesity 2008; 16: 1946-52.

[6] Montgomery-Downs HD, Insana SP, Bond JA. Movement toward a novel activity monitoring device. Sleep Breath 2011; 16(3): 913-7.

[7] Abel MG, Hannon JC, Sell K, Lillie T, Conlin G, Anderson D. Validation of the Kenz Lifecorder EX and ActiGraph GT1M accelerometer for walking and running in adults. Appl Physiol Nutr Metab 2008; 33: 1155-64.

[8] McClain JJ, Sission SB, Tudor-Locke C. Actigraph accelerometer interinstrument reliability during free-living in adults. Med Sci Sports Exerc 2007; 39(9):1509-14

[9] Sasaki JE, John D, Freedson PS. Validation and comparison of ActiGraph activity monitors. J Sci Med Sport 2011; 14: 411-6.

[10] Phillis LRS, Rowlands AV, Parfitt CG. Validation of the genea waveform accelerometer for assessment of children's physical activity. In: Children and Exercise XXVII: The Proceedings of the XXVIIth International Symposium of the European Group of Pediatric Work Physiology. Williams CA, Armstrong N, Eds. Routledge, Abingdon, UK 2011; pp. 133-7.

[11] Tyron WW. The reliability and validity of two ambulatory monitoring actigraphs. Behav Res Methods 2005; 37: 492-7.

[12] FitBit $^{\circledR}$ Inc. c2013. FitBit [online]. Available from $\mathrm{http}: / /$ www.fitbit.com [accessed 5 January 2012].

[13] Freedson PS, Melanson E, Sirard J. Comparison of the Computer Science and Applications, Inc. accelerometer. Med Sci Sports Exerc 1998; 30(5):777-81

[14] Bland JM, Altman DG. Statistical methods for assessing agreement between two methods of clinical measurement. Lancet 1968; 1: 307-10. 